\title{
The 2006 explosion of the recurrent nova RS Ophiuchi
}

T.J. O’Brien ${ }^{* a}$, M.F. Bode ${ }^{b}$, R.W. Porcas ${ }^{c}$, T.W.B. Muxlow ${ }^{a}$, R.J. Beswick ${ }^{a}$, S.T. Garrington $^{a}$, S.P.S. Eyres ${ }^{d}$, J.P. Osborne ${ }^{e}$, K.L. Page ${ }^{e}$, A.P. Beardmore ${ }^{e}$, M.R. Goad ${ }^{e}$, S. Starrfield ${ }^{f}$, J-U. Ness ${ }^{f}$, A. Evans ${ }^{g}$, G.K. Skinner ${ }^{h}$, R.J. Davis ${ }^{a}$

${ }^{a}$ Jodrell Bank Observatory, School of Physics \& Astronomy, University of Manchester, Macclesfield, SK11 9DL, UK

${ }^{b}$ Astrophysics Research Institute, Liverpool John Moores University, Birkenhead, CH41 1LD, UK

${ }^{c}$ Max-Planck-Institut für Radioastronomie, Auf dem Hügel, D-53121 Bonn, Germany

${ }^{d}$ Centre for Astrophysics, University of Central Lancashire, Preston, PRI 2HE, UK

${ }^{e}$ Dept of Physics \& Astronomy, University of Leicester, LE1 7RH, UK

${ }^{f}$ Dept of Physics \& Astronomy, Arizona State University, PO Box 871504, Tempe, AZ 85287-1504, USA

${ }^{g}$ Astrophysics Group, School of Physical \& Geographical Sciences, University of Keele, ST5 $5 B G, U K$

${ }^{h}$ CESR, 31028 Toulouse, France \& Université Paul Sabatier, 31062 Toulouse, France E-mail: tim.obrien@manchester.ac.uk, mfb@astro.livjm.ac.uk, porcas@mpifr-bonn.mpg.de, twbmejb.man.ac.uk, rbeswick@jb.man.ac.uk, stg@jb.man.ac.uk, spseyres@uclan.ac.uk, julo@star.le.ac.uk, kpa@star.le.ac.uk, apb@star.le.ac.uk, mrg@star.le.ac.uk, sumner.starrfield@asu.edu, jan-uwe.nesslasu.edu, aelastro.keele.ac.uk, skinnerlcesr.fr, rjdejb.man.ac.uk

RS Ophiuchi is a recurrent nova comprising a white dwarf accreting material from a companion red giant. After 10-30 years, sufficient material accumulates for a thermonuclear explosion to take place on the white dwarf. The ejected matter slams into the wind of the red giant and sets up shocks which result in copious amounts of radio and X-ray emission. Here, we describe observations of the aftermath of the most recent outburst (discovered 12 Feb 2006) paying particular attention to VLBA/EVN/MERLIN imaging and Swift X-ray spectroscopy.

8th European VLBI Network Symposium

September 26-29, 2006

Toruń, Poland

${ }^{*}$ Speaker. 


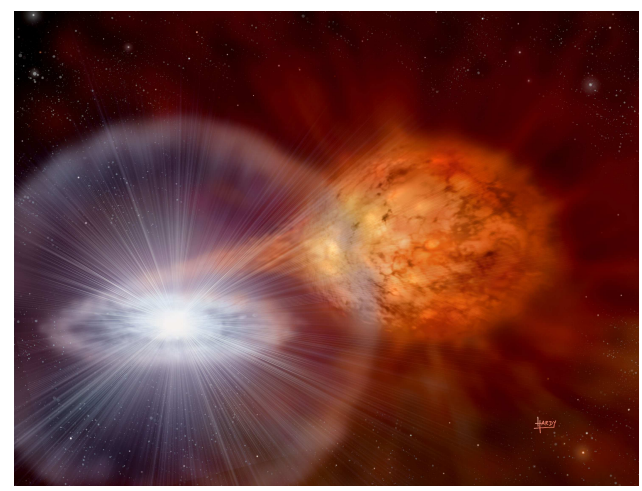

Figure 1: Artist's impression of the RS Oph binary system showing the explosion on the white dwarf which is accreting material from a companion red giant star (David A. Hardy \& PPARC).

\section{Background}

RS Ophiuchi is a recurrent nova system which has been seen to undergo outbursts in 1898, 1933, 1958, 1967 and 1985 (Rosino 1987). Its most recent outburst was discovered on 2006 February 12.83UT (Hirosawa 2006), reaching a peak visual magnitude of 4.5 .

The outbursts result from a thermonuclear explosion in hydrogen-rich material accreted onto the surface of a white dwarf from a companion red giant star (Fig. 1). In this case the white dwarf is thought to be close to the Chandrasekhar limit which, when combined with a supposed high accretion rate, leads to a very short recurrence period compared to that suspected for classical novae (Starrfield et al 1985, Yaron et al 2005). The explosion leads to the ejection of $10^{-6}-10^{-8} \mathrm{M}_{\odot}$ at speeds of around 4,000 $\mathrm{km} \mathrm{s}^{-1}$ (e.g. Buil 2006).

Other than recurrence time, a major difference between RS Oph and classical novae is the presence of a red giant donor rather than a main sequence dwarf star. Following the explosion, the ejected material runs into the wind of the red giant setting up shocks which travel out through the wind and back into the ejecta. Evidence for these shocks was provided by the presence of coronal lines (Rosino 1987) and narrowing of initially broad lines in the optical spectra due to deceleration of material as it sweeps up red giant wind (Snijders 1987, Shore et al 1996).

X-ray observations of the 1985 outburst using EXOSAT (Mason et al 1987) from 55 days after outburst were interpreted as evidence for shock-heated gas with a detection at day 251 as remnant burning on the white dwarf. When combined with radio observations showing a source which peaked in brightness around 40 days after outburst, these allowed the development of a model that led to estimates of the outburst energy, ejected mass, and the density in the red giant wind (Bode \& Kahn 1985, O’Brien \& Kahn 1987, O’Brien et al 1992). In this model, the remnant of RS Oph behaves like that of a supernova remnant but evolving around $10^{5}$ times faster.

The 2006 outburst of RS Oph has been observed extensively at a wide range of wavelengths. Here, we concentrate on radio observations using MERLIN, VLA, VLBA and EVN, and X-ray observations using Swift. 


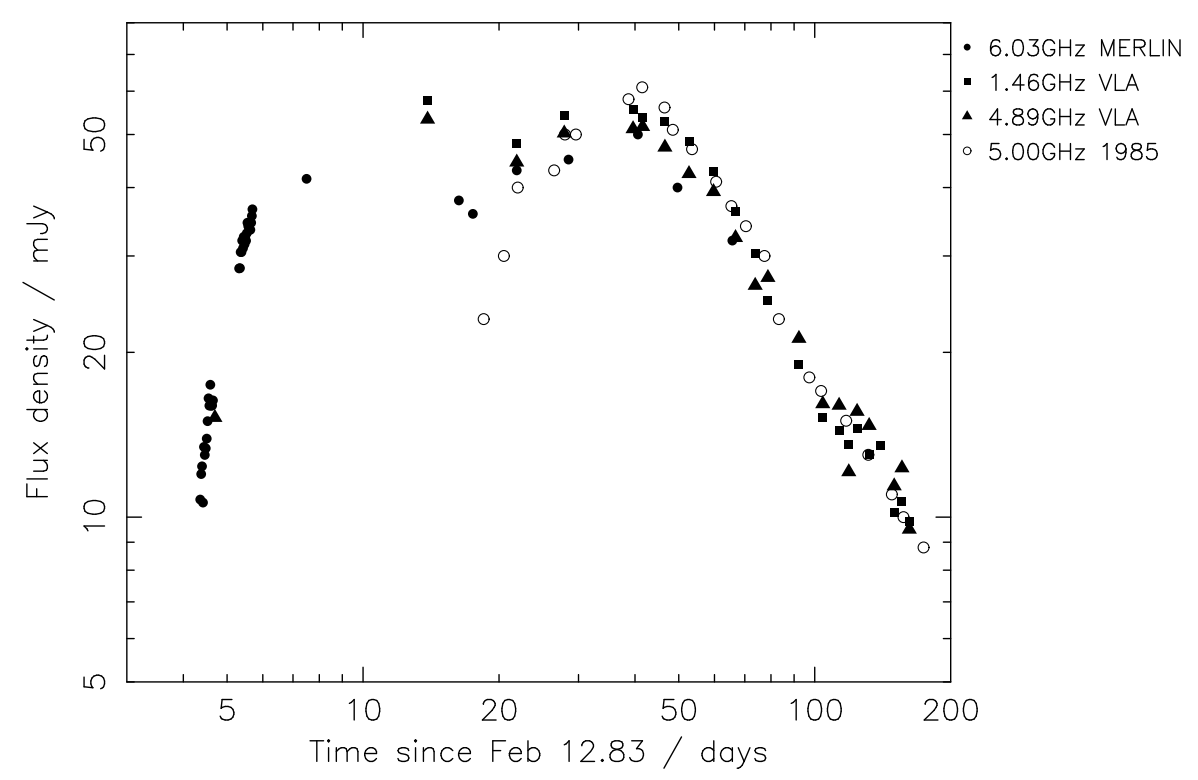

Figure 2: Radio lightcurve of RS Oph using observations with MERLIN and the VLA (from Eyres et al, in prep). Filled symbols are observations from MERLIN (circles) and VLA (squares and triangles) during the 2006 outburst, open circles are observations from the 1985 outburst using Jodrell Bank BBI and VLA (Hjellming et al 1986).

\section{Observations of the 2006 outburst}

\subsection{Radio lightcurve}

Following the discovery that RS Oph had again erupted, we triggered Target of Opportunity observations with MERLIN, the UK's Multi-Element Radio-Linked Interferometer Network. A rapid response meant we were able to begin observations at a frequency of $6 \mathrm{GHz}$ only four days after discovery (Eyres et al 2006, Eyres et al, in prep). Since in 1985 RS Oph was picked up on the rise 18 days after discovery (Padin et al 1985), we were rather surprised to detect it in the first observation at a flux density of $11 \mathrm{mJy}$ and rising rapidly during the 8-hour run, see Fig. 2. A few hours after the first MERLIN observations, the Very Large Array (VLA) measured a consistent flux density of $15 \mathrm{mJy}$ at $4.9 \mathrm{GHz}$.

Following these first observations, the flux density seemed to peak between 8 and 14 days after outburst, decline and then peak again around day 40 , before declining steadily through the remainder of the outburst. A comparison of observations from 1985 and 2006, see Fig. 2, suggests that in 1985 observations did not begin early enough to detect the first peak and only the rise to the second peak was seen. A detailed description of the multifrequency behaviour of the radio lightcurve is provided by Eyres et al (in prep).

\subsection{Radio imaging}

Assuming a distance of $1.6 \pm 0.3 \mathrm{kpc}$ (Bode 1987) and an initial velocity of 4,000 $\mathrm{km} \mathrm{s}^{-1}$, the ejecta will have an angular diameter of around 20 mas one week after outburst. Although this is likely to be an overestimate since we expect deceleration of the ejecta due to interaction with the red giant wind, the implied high brightness temperatures and size scales suggest that VLBI 


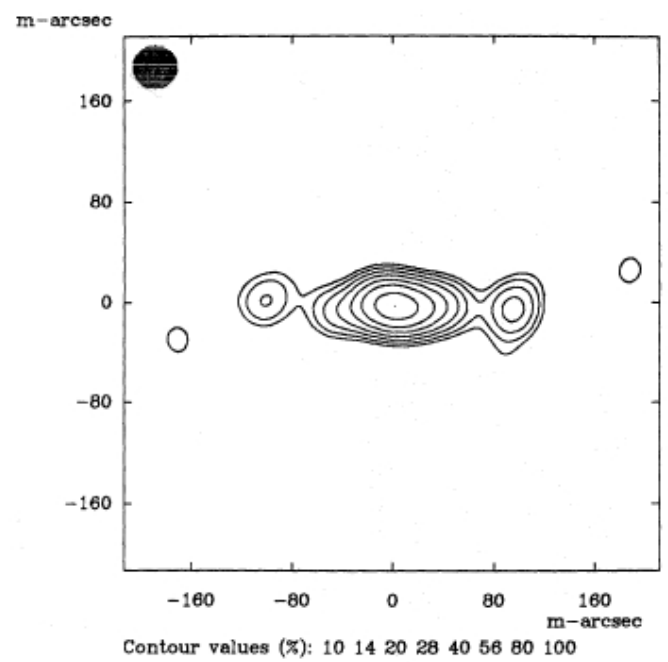

Figure 3: EVN map of RS Oph at $1.7 \mathrm{GHz}$ from 13 Apr 1985 (Taylor et al 1989). The resolution is 35 mas and the peak flux density is $8.8 \mathrm{mJy}$.

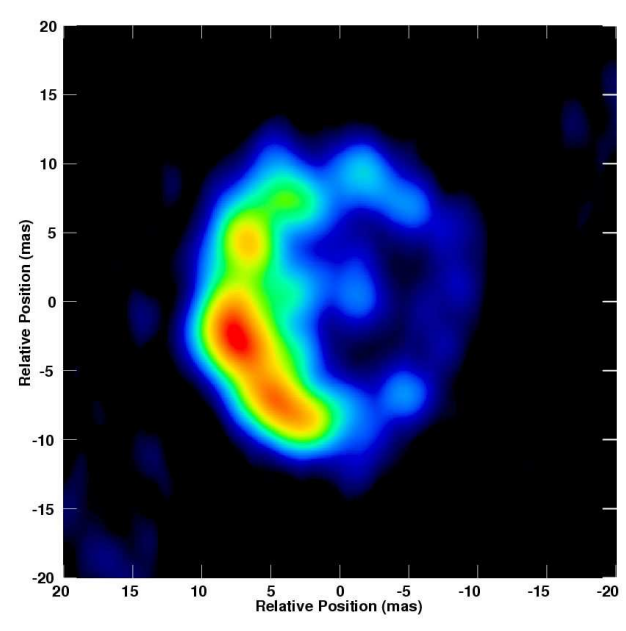

Figure 4: VLBA image of RS Oph at $6 \mathrm{~cm}$ from 26 Feb 2006, 13.8 days after outburst (O'Brien et al 2006). Peak flux density is $4.7 \mathrm{mJybeam}^{-1}$ and the resolution is 3.3 mas.

observations with a resolution of a few mas have the potential to directly image the ejecta soon after outburst.

In 1985, two attempts were made to obtain observations with telescopes in the early European VLBI Network (Porcas et al 1987; Taylor et al 1989). The first observation, 41 days after outburst, was made with a single baseline of Jodrell Bank MkII - Effelsberg. Although the results were not conclusive, a good fit to the visibilities was obtained with a model in which about half the total flux density of $60 \mathrm{mJy}$ was in a core of diameter around 8 mas and the remaining flux resolved out in a larger halo. In the second observation, 77 days after outburst, fringes on the baselines between Effelsberg, Jodrell Bank and Westerbork enabled a map to be made, see Fig. 3. This suggests an east-west structure extending over 200 mas with most of the flux in a central component with a major axis of 60 mas. Taylor et al concluded that the radio emission comprised two components, a low-frequency, non-thermal component which dominates at early times, and a thermal component, visible at higher frequencies, which peaks later and remains optically thick for about 200 days.

Target of Opportunity observations of the 2006 outburst of RS Oph were scheduled with both EVN and the Very Long Baseline Array (VLBA). Triggered by the rapidly rising flux density seen in the MERLIN observations, the first VLBA imaging was carried out 14 days after outburst at wavelengths of 6 and $18 \mathrm{~cm}$. This was followed a week later by EVN imaging at the same wavelengths, and then three more epochs of VLBA imaging 29, 49 and 63 days after outburst (O'Brien at al 2006; O'Brien et al, in prep). Fig. 4 shows the first VLBA image and Fig. 5 the first five epochs of VLBI imaging.

The first VLBA image, Fig. 4, shows an almost circular 17 mas diameter ring of emission, significantly brighter on the eastern side. The peak brightness temperature is around $4 \times 10^{7} \mathrm{~K}$, consistent with claims of non-thermal emission made from the 1985 observations. At 14 days after outburst and assuming a distance of $1.6 \mathrm{kpc}$, the diameter implies an expansion velocity of around $1,700 \mathrm{~km} \mathrm{~s}^{-1}$. This is the first direct image of the shock wave resulting from the explosion. 


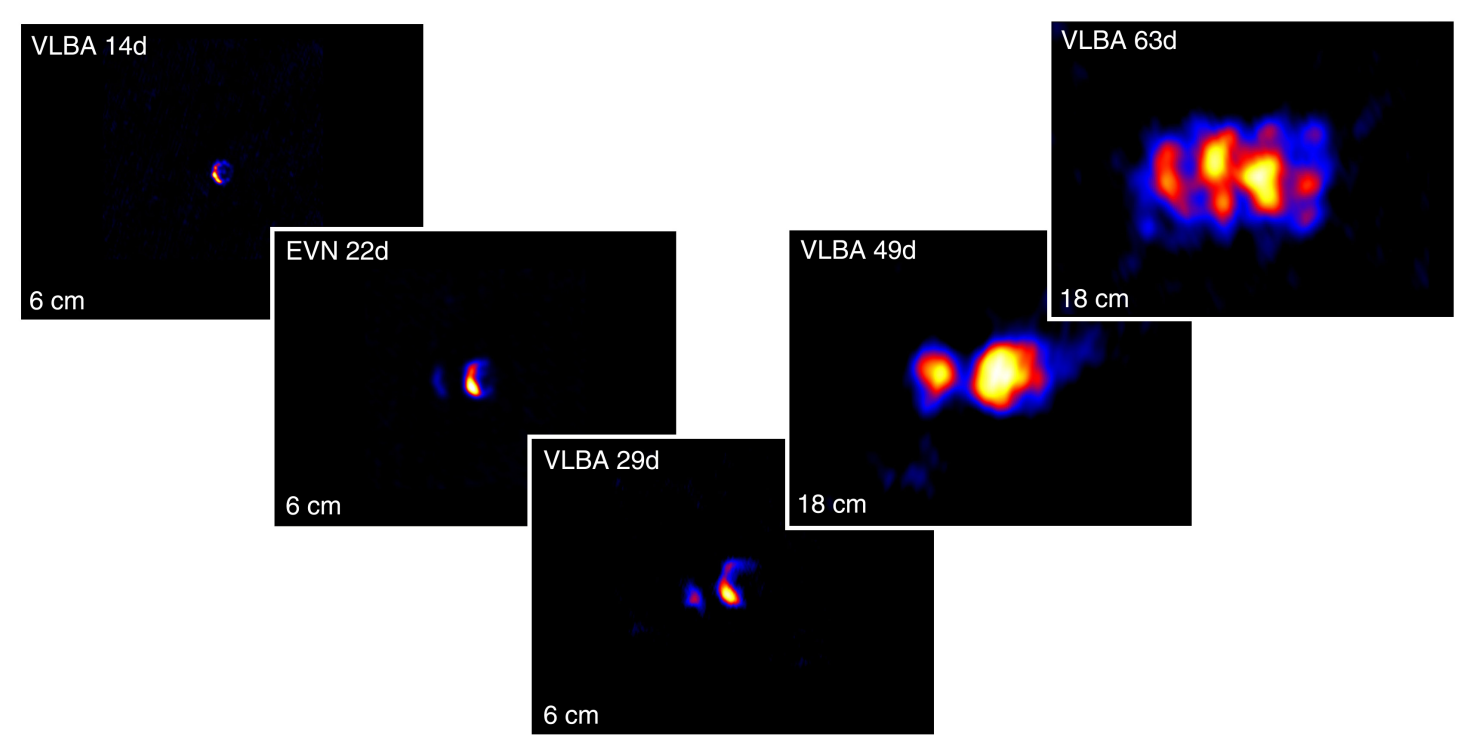

Figure 5: Sequence of VLBI images of RS Oph (O'Brien et al, in prep). Each image is 250 mas in height.

In the following two epochs, see Fig. 5, only the eastern half of the ring is visible expanding almost linearly (see O'Brien et al 2006). Notably, in the second epoch image made with EVN, a component to the east of the ring becomes visible at $6 \mathrm{~cm}$ but not at $18 \mathrm{~cm}$. The following image shows it at both wavelengths. Subsequent imaging with VLBA and MERLIN show a further component appearing to the west of the ring and the source developing into an east-west structure reminiscent of that seen in 1985 (Fig. 3). O'Brien et al (2006) interpret this as evidence for a bipolar shocked shell orthogonal to the plane of the binary orbit, seen at an angle to the line of sight and subject to free-free absorption by the overlying red giant wind.

\subsection{Swift X-ray observations}

The Swift satellite has regularly observed the 2006 explosion of RS Oph from its optical discovery until the time of writing almost 250 days after outburst. The Burst Alert Telescope (BAT) detected X-ray emission in the 14-25 keV band at the time of optical discovery and for several days afterwards whilst the X-Ray Telescope (XRT) has monitored the emission from day 3.17 onwards (Bode et al 2006). In the first 26 days, the spectra were modelled with thermal emission from gas at temperatures of $\sim 10^{7}-10^{8} \mathrm{~K}$ with temporal behaviour consistent with our simple shock models, and in particular the shock wave seen in our radio imaging. Subsequent X-ray observations show the emergence of a supersoft X-ray source (Osborne et al 2006a) which we interpret as evidence of continued nuclear burning of material remaining on the white dwarf. This supersoft phase lasted about 60 days (Osborne et al 2006b) before fading and leaving behind residual emission from the cooling shock-heated red giant wind.

\section{Conclusion}

The first direct images of the shock wave resulting from the explosion of RS Ophiuchi provide an elegant confirmation of the basic model developed following the 1985 outburst. The radio 
imaging and early X-ray observations are consistent with a shock moving at around 1,700 km/s as it expands through the wind of the red giant. However, the detection of the supersoft source shows that, as suggested by O'Brien et al (1992), the interpretation of the EXOSAT observations in 1985 as being solely due to shock-heated gas is likely to be incorrect. The 2006 observations also show that, unlike existing models, the remnant develops into an extremely bipolar structure. It is so far unclear whether this is a result of bipolar ejection i.e. jets, or shaping of an initially more spherical explosion perhaps by an asymmetric red giant wind.

The success of this programme shows the power of observing facilities which are able to respond rapidly and be scheduled flexibly. We are extremely grateful to the directors and staff of the various telescopes in the European VLBI Network, the National Radio Astronomy Observatory and the Swift Mission Operations Center for their generous allocations of observing time.

\section{References}

[1] Rosino, L., in RS Ophiuchi (1985) and the recurrent Nova Phenomenon, ed. M.F. Bode, p1, pub. VNU Science Press, Utrecht (1987)

[2] Hirosawa, K., IAU Circular 8671 (2006)

[3] Starrfield, S., Sparks, W.M., Truran, JW., ApJ, 291, 136 (1985)

[4] Yaron, O., et al, ApJ, 623, 398 (2005)

[5] Buil, C., Central Bureau Electronic Telegram (CBET) 403 (2006)

[6] Snijders, M.A.J., in RS Ophiuchi (1985) and the recurrent Nova Phenomenon, ed. M.F. Bode, p51, pub. VNU Science Press, Utrecht (1987)

[7] Shore, S., et al, ApJ, 456, 717 (1996)

[8] Mason, K.O., et al, in RS Ophiuchi (1985) and the recurrent Nova Phenomenon, ed. M.F. Bode, p167, pub. VNU Science Press, Utrecht (1987)

[9] Bode, M.F., Kahn, F.D., MNRAS, 217, 205 (1985)

[10] O’Brien, T.J., Kahn, F.D., MNRAS, 228, 277 (1987)

[11] O’Brien, T.J., Bode, M.F., Kahn, F.D., MNRAS, 255, 683 (1992)

[12] Hjellming, R.J., et al, ApJ, 305, L71 (1986)

[13] Padin, S., Davis, R.J., Bode, M.F., Nature, 315, 306 (1985)

[14] Eyres, S.P.S., et al, IAU Circular 8678 (2006)

[15] Bode, M.F., in RS Ophiuchi (1985) and the recurrent Nova Phenomenon, ed. M.F. Bode, p241, pub. VNU Science Press, Utrecht (1987)

[16] Porcas, R.W., Davis, R.J., Graham, D.A., in RS Ophiuchi (1985) and the recurrent Nova Phenomenon, ed. M.F. Bode, p203, pub. VNU Science Press, Utrecht (1987)

[17] Taylor, A.R., et al, MNRAS, 237, 81 (1989)

[18] O’Brien, T.J., et al, Nature, 442, 279 (2006)

[19] Bode, M.F., et al, ApJ, in press, astro-ph/0604618 (2006)

[20] Osborne, J., et al, Astronomer's Telegram 770 (2006a)

[21] Osborne, J., et al, Astronomer's Telegram 838 (2006b) 